\title{
On Anti Fuzzy Ideals in Left Almost Semigroups
}

\author{
Madad Khan \\ Department of Mathematics \\ COMSATS Institute of Information Technology \\ Abbottabad, Pakistan \\ E-mail: madadmath@yahoo.com \\ M. Faisal Iqbal \\ Department of Mathematics \\ COMSATS Institute of Information Technology \\ Abbottabad, Pakistan \\ E-mail: fasi_g88@yahoo.com \\ M. Nouman A. Khan \\ Department of Mathematics \\ COMSATS Institute of Information Technology \\ Islamabad, Pakistan \\ E-mail: mailnouman@yahoo.com
}

\begin{abstract}
In this paper we have studied the concept of Anti fuzzy ideals in Left Almost Semigroups (LA-semigroup in short). The equivalent statement for an LA-semigroup to be a commutative semigroup is proved. The set of all anti fuzzy left ideals, which are idempotents, forms a commutative monoid. Moreover it has been shown that the union of any family of Anti fuzzy left ideals of an LA-semigroup is an anti fuzzy left ideal of $F(S)$. The relation of anti fuzzy left(right) ideals, anti fuzzy interior ideals and anti fuzzy bi-ideals in LA-semigroups has been studied. Anti fuzzy points have been defined in an LA-semigroup and has been shown the representation of largest fuzzy left ideal generated by a fuzzy point.
\end{abstract}

Keywords: LA-semigroup, Anti fuzzy ideal, Anti fuzzy point

\section{Introduction}

An LA-semigroup (Kazim, M. A. 1972), is a groupoid $S$ whose elements satisfy the left invertive law,

$$
(a b) c=(c b) a \text { for all } a, b \text { and } c \text { in } S .
$$

An LA-semigroups is a midway between a groupoid and a commutative semigroup. P. Holgate (1992) has explored this useful non-associative structure and called it as simple invertive groupoid. It has wide applications in theory of flocks (Naseeruddin, N. 1970). In an LA-semigroups the medial law (Kazim, M. A. 1972),

$$
(a b)(c d)=(a c)(b d) \text { holds for all } a, b, c \text { and } d \text { in } S .
$$

If there exists an element $e$ in an LA-semigroups $S$ such that $e x=x$ for all $x$ in $S$ then $S$ is called an LA-semigroups with left identity $e$. It is to be noted that if LA-semigroups $S$ contains a right identity then it becomes commutative monoid. If an LA-semigroups $S$ contains left identity then paramedial law,

$$
(a b)(c d)=(d c)(b a) \text { holds for all } a, b, c \text { and } d \text { in } S .
$$

Also $a(b c)=b(a c)$ holds for all $a, b$ and $c$ in an LA-semigroups with left identity. In an LA-semigroups $S$, an element $a \in S$ is called idempotent if $a^{2}=a$.

Rosenfeld was the first who studied fuzzy sets in the structure of groups (Rosenfeld, A. 1971). Kuroki (1981) has studied the bi-ideals in semigroups. A fuzzy subset $f$ of a set $S$ is a function from $S$ to a closed interval $[0,1]$. The concept of a fuzzy set was introduced by Zadeh in 1965. Anti fuzzy bi-ideals have been studied in semigroups in (Shabir, M. 2009). Here in this paper we have generalized the concept of anti fuzzy ideals for LA-semigroups and proved some interesting results. Some preliminaries are given below. 
Let $F(S)$ denote the collection of all fuzzy subsets of an LA-semigroup $S$. For subsets $A, B$ of $S, A B=\{a b \in S: a \in$ $A, b \in B\}$. A non-empty subset $A$ of $S$ is called left(right) ideal of $S$ if $S A \subseteq A(A S \subseteq A)$. Further $A$ is called two-sided ideal if it is both left and right ideal of $S$. A non-empty subset $A$ of $S$ is called an interior ideal of $S$ if $(S A) S \subseteq A$. A non-empty LA-subsemigroup $A$ of $S$ is called bi-ideal of $S$ if $(A S) A \subseteq A$. A non-empty subset $A$ of $S$ is called idempotent if $A A=A$.

Let $f$ and $g$ be two fuzzy subsets of an LA-semigroup $S$. The anti product $f * g$ is defined by

$$
(f * g)(x)= \begin{cases}\bigwedge_{x=y z}\{f(y) \vee g(z)\} ; & \text { if } \exists y \text { and } z \in S, \text { such that } x=y z \\ 1 & \text { otherwise. }\end{cases}
$$

A fuzzy subset of $S$ is called an anti fuzzy LA-subsemigroup of $S$ if $f(a b) \leq f(a) \vee f(b)$ for all $a$ and $b$ in $S$, and is called an anti fuzzy left(right) ideal of $S$ if $f(a b) \leq f(b)(f(a b) \leq f(a))$ for all $a$ and $b$ in $S$. A fuzzy subset $f$ of $S$ is called an anti fuzzy two sided ideal(or an anti fuzzy ideal) of $S$ if it is both anti fuzzy left and anti fuzzy right ideal of $S$. A fuzzy subset $f$ of an LA-semigroup $S$ is called a fuzzy bi-ideal of $S$ if $f((x y) z) \leq f(x) \vee f(z)$ for all $x, y$ and $z$ of $S$.

A fuzzy subset $f$ of an LA-semigroup $S$ is called an anti fuzzy interior ideal of $S$ if $f((x a) y) \leq f(a)$ for all $x, a$ and $y$ of $S$. It can be easily seen that, if $A$ is a non-empty subset of an LA-semigroup $S$, then $A$ is an interior ideal of $S$ if and only if $C_{A^{c}}$ is an anti fuzzy interior ideal of $S$. A fuzzy subset $f$ of $S$ is called an anti fuzzy idempotent if $f * f=f$. For a subset $A$ of $S$ the anti characteristic function, $C_{A^{c}}$ is defined by $C_{A^{c}}=\{.0$ if $x \in A, 1$ if $x \notin A$.

Note that an LA-semigroup $S$ can be considered as an anti fuzzy subset of itself (denoted by $\Theta$ ) and we write $\Theta(x)=0$, for all $x$ in $S$. Let $a$ be an arbitrary element of $S$, then for $\lambda$ in $[0,1)$ and for $x$ in $S$ we define anti fuzzy point $a_{\lambda}$ of $S$ as; $a_{\lambda}(x)=\{. \lambda$ if $x=a, 1$ otherwise..

Proposition 1. Let $S$ be an LA-semigroup, then the set $(F(S), *)$ is an LA-semigroup.

proof. Clearly $F(S)$ is closed. Let $f, g$ and $h$ be in $F(S)$. Let $x$ be any element of $S$ such that it is not expressible as product of two elements in $S$ then we have, $((f * g) * h)(x)=1=((h * g) * f)(x)$. Let the element $x$ can be written as product of two elements in $S$ then we have,

$$
\begin{aligned}
((f * g) * h)(x) & =\bigwedge_{x=y z}\{(f * g)(y) \vee h(z)\} \\
& =\bigwedge_{x=y z}\left\{\bigwedge_{y=p q}\{f(p) \vee g(q)\} \vee h(z)\right\} \\
& =\bigwedge_{x=(p q) z}\{f(p) \vee g(q) \vee h(z)\} \\
& =\bigwedge_{x=(z q) p}\{h(z) \vee g(q) \vee f(p)\} \\
& =\bigwedge_{x=w p}\left\{\bigwedge_{w=z q}(h(z) \vee g(q) \vee f(p))\right\} \\
& =\bigwedge_{x=w p}\{(h * g)(w) \vee f(p)\} \\
& =((h * g) * f)(x) .
\end{aligned}
$$

Hence $(F(S), *)$ is an LA-semigroup.

Corollary 1. Let $S$ be an LA-semigroup, then the medial law holds in $F(S)$.

proof. Let $f, g, h$, and $k$ be arbitrary elements of $F(S)$. By successive use of left invertive law, $(f * g) *(h * k)=$ $((h * k) * g) * f=((g * k) * h) * f=(f * h) *(g * k)$.

Theorem 1. Let $S$ be an LA-semigroup with left identity, then the following properties hold in $F(S)$;

(i) $\quad f *(g * h)=g *(f * h) \quad$ for all $f, g$ and $h$ in $F(S)$,

(ii) $\quad(f * g) *(h * k)=(k * h) *(g * f) \quad$ for all $f, g, h$ and $k$ in $F(S)$.

proof. (i) Let $x$ be an arbitrary element of $S$. If $x$ is not expressible as a product of two elements in $S$, then $(f *(g * h))(x)=$ 
$1=(g *(f * h))(x)$. Let there exists $y$ and $z$ in $S$ such that $x=y z$, then

$$
\begin{aligned}
(f *(g * h))(x) & =\bigwedge_{x=y z}\{f(y) \vee(g * h)(z)\} \\
& =\bigwedge_{x=y z}\left\{f(y) \vee \bigwedge_{z=p q}\{g(p) \vee h(q)\}\right\} \\
& =\bigwedge_{x=y(p q)}\{f(y) \vee g(p) \vee h(q)\} \\
& =\bigwedge_{x=p(y q)}\{g(p) \vee f(y) \vee h(q)\} \\
& =\bigwedge_{x=p w}\left\{g(p) \vee \bigwedge_{w=y q}\{f(y) \vee h(q)\}\right\} \\
& =\bigwedge_{x=p w}\{g(p) \vee(f * h)(w)\} \\
& =(g *(f * h))(x) .
\end{aligned}
$$

Thus, $(f *(g * h))(x)=(g *(f * h))(x)$. If $z$ is not expressible as a product of two elements in $S, \operatorname{then}(f *(g * h))(x)=$ $1=(g *(f * h))(x)$. Hence, $(f *(g * h))(x)=(g *(f * h))(x)$ for all $x$ in $S$.

(ii) If any element $x$ of $S$ is not expressible as product of two elements in $S$ at any stage, $\operatorname{then}((f * g) *(h * k))(x)=1=$ $((k * h) *(g * f))(x)$. Let there exists $y, z$ in $S$ such that $x=y z$, then

$$
\begin{aligned}
((f * g) *(h * k))(x) & =\bigwedge_{x=y z}\{(f * g)(y) \vee(h * k)(z)\} \\
& =\bigwedge_{x=y z}\left\{\bigwedge_{y=p q}\{f(p) \vee g(q)\} \vee \bigwedge_{z=u v}\{h(u) \vee k(v)\}\right\} \\
& =\bigwedge_{x=(p q)(u v)}\{f(p) \vee g(q) \vee h(u) \vee k(v)\} \\
& =\bigwedge_{x=(v u)(q p)}\{k(v) \vee h(u) \vee g(q) \vee f(p)\} \\
& =\bigwedge_{x=m n}\left\{\bigwedge_{m=v u}\{k(v) \vee h(u)\} \vee \bigwedge_{n=q p}\{g(q) \vee f(p)\}\right\} \\
& =\bigwedge_{x=m n}\{(k * h)(m) \vee(g * f)(n)\} \\
& =((k * h) *(g * f))(x) .
\end{aligned}
$$

Proposition 2. An LA-semigroup $S$ with $F(S)=(F(S))^{2}$ is commutative semigroup if and only if $(f * g) * h=f *(h * g)$ holds for all fuzzy subsets $f, g$ and $h$ of $S$.

proof. It is simple.

Lemma 1. A non-empty subset $A$ of an LA-semigroup $S$ is LA-subsemigroup if and only if $C_{A^{c}}$ is an anti fuzzy LAsubsemigroup of $S$.

proof. Let $A$ be a non-empty subset of an LA-semigroup $S$ and $x$ and $y$ be arbitrary elements of $S$. Let $A$ be an LAsubsemigroup of $S$. Let $x$ and $y$ be in $A$ then $x y$ is also in $A$, so we have,

$$
C_{A^{c}}(x y)=0=C_{A^{c}}(x) \vee C_{A^{c}}(y) .
$$

Now let $x$ be in $A$ and $y$ in not in $A$ then $C_{A^{c}}(x)=0$ and $C_{A^{c}}(y)=1$ and so we have,

$$
C_{A^{c}}(x y) \leq 1=C_{A^{c}}(x) \vee C_{A^{c}}(y) .
$$

Now let both $x$ and $y$ are not in $A$ then $C_{A^{c}}(x)=1$ and $C_{A^{c}}(y)=1$ and so we have,

$$
C_{A^{c}}(x y) \leq 1=C_{A^{c}}(x) \vee C_{A^{c}}(y) .
$$

Thus for all $x$ and $y$ in $S$ we have $C_{A^{c}}(x y) \leq C_{A^{c}}(x) \vee C_{A^{c}}(y)$, which implies that $C_{A^{c}}$ is an anti fuzzy LA-subsemigroup of $S$. 
Conversely, let $C_{A^{c}}$ be an anti fuzzy LA-subsemigroup of $S$. If the elements $x$ and $y$ are in $A$ then $C_{A^{c}}(x)=0=C_{A^{c}}(y)$. But $C_{A^{c}}(x y) \leq C_{A^{c}}(x) \vee C_{A^{c}}(y)=0$, which implies that $x y$ is in $A$. Hence $A$ is an LA-subsemigroup of $S$.

Lemma 2. A non-empty subset A of an LA-semigroup $S$ left(right, two-sided) ideal of $S$ if and only if $C_{A^{c}}$ is an anti fuzzy left(right, two-sided) ideal of $S$.

proof. Let $A$ be a non-empty subset of an LA-semigroup $S$ and is an anti fuzzy left ideal. Let $x$ and $y$ be arbitrary elements of $S$ such that both $x$ and $y$ are in $A$. Then since $A$ is left ideal so $x y$ is also in $A$. Thus we have,

$$
C_{A^{c}}(x y)=0=C_{A^{c}}(y) .
$$

Now let $x$ be in $A$ and $y$ in not in $A$ then $C_{A^{c}}(x)=0$ and $C_{A^{c}}(y)=1$ and so we have,

$$
C_{A^{c}}(x y) \leq 1=C_{A^{c}}(y) .
$$

Now let both $x$ and $y$ are not in $A$ then $C_{A^{c}}(x)=1$ and $C_{A^{c}}(y)=1$ and so we have,

$$
C_{A^{c}}(x y) \leq 1=C_{A^{c}}(y) .
$$

Thus for all $x$ and $y$ in $S$ we have $C_{A^{c}}(x y) \leq C_{A^{c}}(y)$, which implies that $C_{A^{c}}$ is an anti fuzzy left ideal of $S$.

Conversely, let $C_{A^{c}}$ be an anti fuzzy left ideal of $S$. If the elements $x$ and $y$ are in $A$ then $C_{A^{c}}(x)=0=C_{A^{c}}(y)$. But $C_{A^{c}}(x y) \leq C_{A^{c}}(y)=0$, which implies that $x y$ is in $A$. Hence $A$ is left ideal of $S$.

Similarly we can prove the result for right and two sided ideal of $S$.

For non-empty subsets $A$ and $B$ of an LA-semigroup $S, C_{A} * C_{B}=C_{A B}$. It is easy to see that for every fuzzy subset $f$ of an LA-semigroup $S$, we have $f \subseteq S$. The following lemmas have the same proof as in (Holgate, P. 1992).

Lemma 3. Let $f$ be a fuzzy subset of an LA-semigroup $S$, then the following properties hold.

(i) $f$ is an anti fuzzy LA-subsemigroup of $S$ if and only if $f * f \supseteq f$.

(ii) $f$ is an anti fuzzy left ideal of $S$ if and only if $\Theta * f \supseteq f$.

(iii) $f$ is an anti fuzzy right ideal of $S$ if and only if $f * \Theta \supseteq f$.

(iv) $f$ is an anti fuzzy ideal of $S$ if and only if $\Theta * f \supseteq f$ and $f * \Theta \supseteq f$. proof. It is same as in (Shabir, M. 2009).

Lemma 4. Let $S$ be an LA-semigroup. Then the following properties hold.

(i) Let $f$ and $g$ be two anti fuzzy LA-subsemigroups of $S$. Then $f \cup g$ is also an anti fuzzy LA-subsemigroup of $S$.

(ii) The union of any family of anti fuzzy left(right, two-sided) ideal of $S$ an anti fuzzy left(right, two-sided) ideal of $S$.

proof. (i) Let $f$ and $g$ be two anti fuzzy LA-subsemigroups of $S$ and $x, y$ be any two arbitrary elements of $S$, then $(f \cup g)(x y)=f(x y) \vee g(x y) \leq f(x) \vee f(y) \vee g(x) \vee g(y)=f(x) \vee g(x) \vee f(y) \vee g(y)$ thus we have $(f \cup g)(x y) \leq$ $(f \cup g)(x) \vee(f \cup g)(y)$. Hence $f \cup g$ is an anti fuzzy LA-subsemigroup.

(ii) Let $\left\{f_{i}\right\}_{i \in I}$ be a family of anti fuzzy left ideals of $S$, for $x$ and $y$ in $S$ we have

$$
\begin{aligned}
\left(\bigcup_{i \in I} f_{i}\right)(x y) & =\bigvee_{i \in I}\left(f_{i}(x y)\right) \\
& \leq \bigvee_{i \in I} f_{i}(y) \\
& =\left(\bigcup_{i \in I} f_{i}\right)(y) .
\end{aligned}
$$

Hence $\bigcup_{i \in I} f_{i}$ is an anti fuzzy left ideal of $S$.

Lemma 5. In an LA-semigroup with left identity $\Theta * \Theta=\Theta$.

proof. Every $x$ in $S$ can be written as $x=e x$, where $e$ is the left identity in $S$. So $(\Theta * \Theta)(x)=\bigwedge_{e x=y z}\{\Theta(y) \vee \Theta(z)\} \leq$ $\{\Theta(e) \vee \Theta(x)\}=0$. Hence $(\Theta * \Theta)(x)=0=\Theta(x)$ for all $x$ in $S$.

Lemma 6. In an LA-semigroup $S$ with left identity, for every anti fuzzy left ideal $f$ of $S$, we have $(\Theta * f)=f$.

proof. It is sufficient to show that $\Theta * f \subseteq f$. Now for any $x$ in $S,(\Theta * f)(x)=\bigwedge_{x=y z}\{\Theta(y) \vee f(z)\}$. Since $x=e x$, for all $x$ in $S$, as $e$ is left identity in $S$, so $\bigwedge_{e x=y z}\{\Theta(y) \vee f(z)\} \leq \Theta(e) \vee f(x)=f(x)$. 
Proposition 3. Let $S$ be an LA-semigroup with left identity and $f$ and $k$ are fuzzy left ideals in $S$ then for any fuzzy subsets $g$ and $h$ of $S, f * g=h * k$ implies that $g * f=k * h$.

proof. Since $f$ and $h$ are fuzzy left ideals in $S$ so by above lemma $4, \Theta * f=f$ and $\Theta * h=h$. Now $g * f=(\Theta * g) * f=$ $(f * g) * \Theta=(h * k) * \Theta=(\Theta * k) * h=k * h$.

The following corollary is direct consequence of the successive use of left invertive law in fuzzy LA-semigroup shown in proposition 1 .

Theorem 2. If $S$ is an LA-semigroup then $Q=\{f \mid f \in \Theta, f * h=f$ where $h=h * h\}$ is a commutative subsemigroup with identity of $S$.

proof. It is simple.

Let $S$ be an LA-semigroup and $a_{\lambda}$ be an anti fuzzy point in $S$. The largest anti fuzzy left ideal of $S$ containing $a_{\lambda}$ is called anti fuzzy left ideal of $S$ generated by $a_{\lambda}$. Let us by $\left\langle a_{\lambda}\right\rangle_{L}$ we shall mean an anti fuzzy left ideal of $S$ generated by $a_{\lambda}$.

Theorem 3. Let $S$ be an LA-semigroup with left identity e and $a_{\lambda}$ be an anti fuzzy point in $S$, then $\left\langle a_{\lambda}\right\rangle_{L}=f$,

$$
\text { wheref }(x)=\left\{\begin{array}{l}
\lambda \in[0,1) \text { if there exists } b \in S \text { such that } x=b a \\
1
\end{array}\right. \text { otherwise. }
$$

proof. Let $x$ and $y$ be arbitrary elements of $S$. Let $f(y)=1$ then $f(x y) \leq 1=f(y)$ and if $f(y) \neq 1$ then there exists an element $b$ in $S$ such that $y=b a$. Now, $f(x y)=f(x(b a))=f((e x)(b a))=f((a b)(x e))=f(((x e) b) a)=\lambda \leq f(y)$, which implies that $f$ is an anti fuzzy left ideal in $S$. Since $a=e a$, so $f(a)=f(e a)=\lambda$ and hence $a_{\lambda}$ is in $f$. Let $g$ be any other anti fuzzy left ideal of $S$ containing $a_{\lambda}$, then $g(a) \leq \lambda$. Let $f(x)=1$ for all $x$ in $S$ then $g(x) \leq 1=f(x)$. On the other hand if $f(x)=\lambda$ then there exists $b$ in $S$ such that $x=b a$ and $g(x)=g(b a) \leq g(a) \leq \lambda=f(x)$, which implies that $g \subseteq f$. Hence $f$ is the largest anti fuzzy left ideal generated by $a$ in $S$.

Proposition 4. Every idempotent anti fuzzy left ideal of an LA-semigroup $S$ is an anti fuzzy ideal of $S$.

proof. Let $f$ be an anti fuzzy left ideal of $S$, which is idempotent. Consider, $f * \Theta=(f * f) * \Theta=(\Theta * f) * f \supseteq f * f=f$.

Theorem 4. Let $f$ is an anti fuzzy idempotent in an LA-semigroup $S$ with left identity, then $\Theta * f$ is an idempotent.

proof. Let $f$ be an anti fuzzy idempotent in $S$, by lemma 3 and using medial law we have $(\Theta * f) *(\Theta * f)=(\Theta * \Theta) *(f * f)=$ $(\Theta * f)$.

Theorem 5. Let $S$ be an LA-semigroup with left identity, then the collection of all anti fuzzy left ideals of $S$, which are idempotent forms a commutative monoid.

proof. Let $\hat{H}$ denote the collection of all anti fuzzy left ideals which are idempotent in $S$. Here, $\hat{H}$ is non-empty, since by lemma $3, \Theta * \Theta=\Theta$ implies that $S$ is in $\hat{H}$. Consider $f, g$ in $\hat{H}$, then $(f * g) *(f * g)=(f * f) *(g * g)=f * g$, also by corollary $1, \Theta *(f * g)=(\Theta * \Theta) *(f * g)=(\Theta * f) *(\Theta * g) \supseteq f * g$. Also for every $f, g$ in $\hat{H}$ by use of theorem 1 and corollary 1, we get $f * g=(f * g) *(f * g)=(g * f) *(g * f)=(g * g) *(f * f)=g * f$, that is commutative law holds in $\hat{H}$. Now, for any $f, g$, and $h$ in $\hat{H}$ we have $(f * g) * h=(h * g) * f=f *(h * g)=f *(g * h)$. Since every $f$ in $\hat{H}$ is an anti left ideal, so lemma 4 implies that $\Theta * f=f$. Commutativity implies that $\Theta * f=f * \Theta=f$, which implies that $S$ is identity in $\hat{H}$ and every $f$ is an anti fuzzy ideal in $\hat{H}$.

Lemma 7. Let $S$ be an LA-semigroup with left identity e, then every fuzzy right ideal is an anti fuzzy ideal.

proof. Let $f$ be an anti fuzzy right ideal in $S$, so $f * \Theta \supseteq f$. By lemma 3 and proposition $1, \Theta * f=(\Theta * \Theta) * f=$ $(f * \Theta) * \Theta \supseteq f * \Theta \supseteq f$. So $f$ is an anti fuzzy left ideal, and hence an anti fuzzy ideal in $S$.

Remark 1. If $f$ is a fuzzy right ideal of an LA-semigroup $S$ with left identity then $f \cup(\Theta * f)$ and $f \cup(f * f)$ are fuzzy two-sided ideals of $S$.

Lemma 8. If $f$ is an anti fuzzy left ideal of an LA-semigroup $S$ with left identity then $f \cup(f * \Theta)$ and $f \cup(f * f)$ are anti fuzzy two-sided ideals of $S$.

proof. Consider, $(f \cup(f * \Theta)) * \Theta=(f * \Theta) \cup((f * \Theta) * \Theta)=(f * \Theta) \cup((\Theta * \Theta) * f)=(f * \Theta) \cup(\Theta * f)=(f * \Theta) \cup f=$ $f \cup(f * \Theta)$. Hence $f \cup(f * \Theta)$ is fuzzy right ideal of $S$, and by lemma $5, f \cup(f * \Theta)$ is fuzzy two-sided ideal of $S$. Now $(f \cup(f * f)) * \Theta=(f * \Theta) \cup(f * f) * \Theta=(f * \Theta) \cup((\Theta * f) * f) \supseteq(f * \Theta) \cup(f * f)=(f * f) \cup(\Theta * f) \supseteq(f * f) \cup f=f \cup(f * f)$, implies that $f \cup(f * f)$ is a fuzzy right ideal of $S$. By lemma 5, $f \cup(f * f)$ is a fuzzy left ideal of $S$.

Theorem 6. A non-empty subset $A$ of an LA-semigroup $S$ is a bi-ideal of $S$ if and only if $C_{A^{c}}$ is an anti fuzzy bi-ideal of $S$.

proof. Let $A$ be a non-empty subset of LA-semigroup $S$ and is a bi-ideal of $S$. Since $A$ is an LA-subsemigroup of $S$, then $C_{A^{c}}$ is an anti fuzzy LA-subsemigroup of $S$. Let $x, y$ and $z$ be arbitrary elements of $S$ such that both $x$ and $z$ are in $A$. Then 
since $A$ is a bi-ideal so $((x y) z)$ is also in $A$. Thus we have,

$$
C_{A^{c}}((x y) z)=0 \leq C_{A^{c}}(x) \vee C_{A^{c}}(z) .
$$

Now let $x$ be not in $A$ and $z$ is in $A$ then $C_{A^{c}}(x)=1$ and $C_{A^{c}}(z)=0$ and so we have,

$$
C_{A^{c}}((x y) z) \leq 1=C_{A^{c}}(x) \vee C_{A^{c}}(z) .
$$

Now let both $x$ and $z$ are not in $A$ then $C_{A^{c}}(x)=1$ and $C_{A^{c}}(z)=1$ and so we have,

$$
C_{A^{c}}((x y) z) \leq 1=C_{A^{c}}(x) \vee C_{A^{c}}(z)
$$

Thus for all $x, y$ and $z$ in $S$ we have $C_{A^{c}}((x y) z) \leq C_{A^{c}}(x) \vee C_{A^{c}}(z)$, which implies that $C_{A^{c}}$ is an anti fuzzy bi-ideal of $S$.

Conversely, let $C_{A^{c}}$ be an anti fuzzy bi-ideal of $S$. Since $C_{A^{c}}$ is the anti fuzzy LA-subsemigroup of $S$ so $A$ is the LAsubsemigroup of $S$. If $a$ belongs to $(A S) A$ that is $a=(x y) z$ for all $x$ and $z$ in $A$ and $y$ in $S$, then $C_{A^{c}}(x)=0=C_{A^{c}}(z)$. But $C_{A^{c}}((x y) z) \leq C_{A^{c}}(x) \vee C_{A^{c}}(z)=0$, which implies that $((x y) z)$ is in $A$. Hence $A$ is bi-ideal of $S$.

Lemma 9. Let $f$ be an anti fuzzy LA-subsemigroup of an LA-semigroup $S$. Then $f$ is a fuzzy bi-ideal of $S$ if and only if $(f * \Theta) * f \supseteq f$.

proof. Let $f$ be an anti fuzzy LA-subsemigroup of $S$, Let $a$ be an arbitrary element of $S$. If there exist $x$ and $y$ in $S$ such that $a=x y$, then

$$
\begin{aligned}
((f * \Theta) * f)(x) & =\bigwedge_{a=x y}\{(f * \Theta)(x) \vee f(y)\} \\
& =\bigwedge_{a=x y}\left\{\bigwedge_{x=p q}\{f(p) \vee \Theta(q)\} \vee f(y)\right\} \\
& =\bigwedge_{a=(p q) y}\{f(p) \vee f(y)\} \\
& \geq f((p q) y)=f(x)
\end{aligned}
$$

which implies $((f * \Theta) * f)(x) \geq f(x)$. If $x$ is not expressible as a product of two elements then $((f * \Theta) * f)(x)=1 \geq f(x)$. Hence $(f * \Theta) * f \supseteq f$.

Conversely, suppose that $(f * \Theta) * f \supseteq f$. Let $x, y$ and $z$ be arbitrary elements of $S$. Then

$$
\begin{aligned}
f((x y) z) & \leq((f * \Theta) * f)((x y) z) \\
& =\bigwedge_{((x y) z)=a b}\{(f * \Theta)(a) \vee f(b)\} \\
& =\bigwedge_{((x y) z)=a b}\left\{\bigwedge_{a=p q}\{f(p) \vee \Theta(q)\} \vee f(b)\right\} \\
& =\bigwedge_{((x y) z)=(p q) b}\{f(p) \vee f(b)\} \\
& \leq f(x) \vee f(z)
\end{aligned}
$$

Hence for all $x, y$ and $z$ in $\Theta, f((x y) z) \leq f(x) \vee f(z)$ that is $f$ is an anti fuzzy bi-ideal of $S$.

Lemma 10. Let $f$ and $g$ be anti fuzzy right ideals of an LA-semigroup $S$ with left identity. Then $f * g$ and $g * f$ are anti fuzzy bi-ideals of $S$.

proof. By corollary 1 , we have $(f * g) *(f * g)=(f * f) *(g * g) \supseteq f * g$. Hence $f * g$ is a fuzzy LA-subsemigroup of $S$. Now, by proposition 1 , lemma 3 and corollary 1 , we have $((f * g) * \Theta)) *(f * g)=((f * g) *(\Theta * \Theta)) *(f * g)=$ $((f * \Theta) *(g * \Theta)) *(f * g) \supseteq(f * g) *(f * g) \supseteq f * g$. Similarly, $g * f$ is a bi-ideal of $S$.

Lemma 11. Let $f$ be an anti fuzzy LA-subsemigroup of an LA-semigroup $S$. Then $f$ is an anti fuzzy interior ideal of $S$ if and only if $(\Theta * f) * \Theta \supseteq f$.

proof. Proof is similar to lemma 9.

Proposition 5. Let $S$ be an LA-semigroup. Then for any anti fuzzy left ideal, which is idempotent, in $S$, the following properties hold.

(i) $f$ is an anti fuzzy bi-ideal. 
(ii) $f$ is an anti fuzzy interior ideal.

proof. (i) Since a fuzzy subset $f$ of $S$ is an anti fuzzy left ideal so $f * f \supseteq f$. By corollary 1 , we get $(f * \Theta) * f=$ $(f * \Theta) *(f * f)=(f * f) *(\Theta * f) \supseteq f * f=f$.

(ii) Consider, $(\Theta * f) * \Theta \supseteq f * \Theta=(f * f) * \Theta=(\Theta * f) * f \supseteq f * f=f$, which implies that $f$ is an anti fuzzy interior ideal of $S$.

Lemma 12. Every fuzzy subset $f$ of an LA-semigroup $S$ with left identity is an anti fuzzy right ideal if and only if it is an anti fuzzy interior ideal.

proof. Let every fuzzy subset $f$ of $S$ is an anti fuzzy right ideal. For $x, a$ and $y$ of $S$, consider $f((x a) y) \leq f(x a)=$ $f((e x) a)=f((a x) e) \leq f(a x) \leq f(a)$, which implies that $f$ is an interior ideal. Conversely, for any $x$ and $y$ in $S$ we have, $f(x y)=f((e x) y) \leq f(x)$.

Lemma 13. Let $f$ be an anti fuzzy left ideal in an LA-semigroup $S$ with left identity, then $f$ being anti fuzzy interior ideal is an anti fuzzy bi-ideal of $S$.

proof. Since $f$ is an anti fuzzy left ideal in $S$, so $f(x y) \leq f(y)$ for all $x$ and $y$ in $S$. As $e$ is left identity in $S$. So, $f(x y)=f((e x) y) \leq f(x)$, which implies that $f(x y) \leq f(x) \vee f(y)$ for all $x$ and $y$ in $S$. Thus $f$ is an anti fuzzy LAsubsemigroup of $S$. For any $x, y$ and $z$ in $S$, we get $f((x y) z)=f((x(e y)) z)=f((e(x y)) z) \leq f(x y)=f((e x) y) \leq f(x)$. Also $f((x y) z)=f((z y) x)=f((z(e y)) x)=f((e(z y)) x) \leq f(z y)=f((e z) y) \leq f(z)$. Hence $f((x y) z) \leq f(x) \vee f(z)$ for all $x, y$ and $z$ in $S$.

Proposition 6. Let $f$ is a fuzzy subset of an LA-semigroup $S$ with left identity. If $f$ is an anti fuzzy left(right, two-sided) ideal in $S$ then $f * f$ is an anti fuzzy ideal in $S$.

proof. Let $f$ be an anti fuzzy left ideal in an LA-semigroup $S$, then by lemma 1 , we have $S * f \supseteq f$. By use of lemma 3 and corollary $1, \Theta *(f * f)=(\Theta * \Theta) *(f * f)=(\Theta * f) *(\Theta * f) \supseteq f * f$. Also by proposition $1,(f * f) * \Theta=(\Theta * f) * f \supseteq f * f$. If $f$ is an anti fuzzy right ideal in $S$ then by lemma $5, f$ is an anti fuzzy left ideal of $S$.

Corollary 2. Let $f$ is an anti fuzzy subset of an LA-semigroup $S$ with left identity. If $f$ is an anti fuzzy left ideal in $S$ then $f * f$ is an anti fuzzy bi-ideal and an anti fuzzy interior ideal of $S$.

proof. By proposition $6, f * f$ is an anti fuzzy ideal in $S$. Now by lemmas 11 and $12, f * f$ is an anti fuzzy interior and an anti fuzzy bi-ideal of $S$.

Theorem 7. In an LA-semigroup $S$, every anti fuzzy ideal is an anti fuzzy bi-ideal and an anti fuzzy interior ideal of $S$.

proof. Let $f$ be an anti fuzzy ideal of an LA-semigroup $S$. Clearly $f$ is an LA-subsemigroup of $S$ by lemma 1 , since $f * f \supseteq f$. Consider, $(f * \Theta) * f \supseteq f * f \supseteq f$, which by lemma 7, shows that $f$ is a fuzzy bi-ideal of $S$. Now, consider $(\Theta * f) * \Theta \supseteq f * \Theta \supseteq f$, which by lemma 10 shows that $f$ is a fuzzy interior ideal of $S$.

\section{References}

Holgate, P. (1992). Groupoids satisfying a simple invertive law. The Math. Stud., 61, 101-10.

Kazim, M. A. and M. Naseeruddin. (1972). On LA-semigroup. Allig. Bull. Math., 8, 1-7.

Kuroki, N. (1979). Fuzzy bi-ideals in Semigroups. Comment. Math. Univ. St. Pauli, 27, 17-21.6.

Kuroki, N. (1981). On fuzzy ideals and fuzzy bi-ideals in semigroups. Fuzzy Sets and Systems, 5 203-215.

Mordeson, J. N. (2003). Fuzzy semigroups, Springer-Verlag, Berlin.

Mushtaq, Q. and Madad Khan. (2006). Ideals in AG-band and AG*-groupoid, Quasigroups and Related Systems, 14, 207-215.

Naseeruddin, N. (1970). Some studies in almost semigroups and flocks, Ph.D. thesis, Aligarh Muslim University, Aligarh, India.

Protic, P. V. and N. Stevanović. (1994). On Abel-Grassmann's groupoids. Proc. Conf. Pristina, 31-38.

Rosenfeld, A. (1971). Fuzzy groups. J. Math. Anal. Appl., 35, 512-517.

Shabir, M. (2005). Fully fuzzy prime semigroups. Inter. J. Math. and Math. Sci., 163-168.

Shabir, M. and Y. Nawaz. (2009). Semigroups characterized by the properties of their anti fuzzy ideals. Journal of advance Research in Pure Mathematics, 3, 42-59.

Xiang-Yun Xie. (2001). On prime, quasi-prime, weakly quasi-prime fuzzy left ideals of semigroups. Fuzzy Sets and Systems, 123, 239-249.

Xiang-Yun Xie and Jian Tang. (2008). Fuzzy radicals and prime fuzzy ideals of ordered semigroups. Inform. Sci., 178, 
4357-4374.

Wang Xue-ping, Mo Zhi-wen and Liu Wang-jin. (1992). Fuzzy Ideals Generated by Fuzzy point in Semigroup. J. Sichuan Normal Univ., 15 17-24.

Zadeh, L.A. (1965). Fuzzy sets. Inform. Control, 8, 338-353. 